\title{
Adoptive Immunotherapy
}

National Cancer Institute

\section{Source}

National Cancer Institute. Adoptive Immunotherapy. NCI Thesaurus. Code C15351.

A technique for enhancing cell-mediated immunity by: 1) artificially increasing the number of specific immunocompetent cells through inoculation of either ex-vivo expanded primarily activated cytotoxic lymphocytes or genetically engineered lymphocytes with enhanced cytotoxic ability or 2 ) by altering tumor cells to make them more immunogenic. 